\title{
A interface entre o ensino do processo de enfermagem e sua aplicação na prática assistencial
}

\section{The interface between teaching of the nursing process and the application in professional practice}

\section{La interfaz entre la enseñanza del proceso de enfermería y su aplicación en la práctica asistencial}

\author{
Lúcia Nazareth Amante', Jane Cristina Anders", Betina H. S. Meirelles"', \\ Maria Itayra Padilha'v , Denise Faucz Kletemberg ${ }^{\mathrm{V}}$
}

\footnotetext{
'Enfermeira. Doutora em Enfermagem. Docente do Departamento de Enfermagem (NFR) da Universidade Federal de Santa Catarina (UFSC).

Florianópolis, SC. E-mail: luciamante@ccs.ufsc.br.

"Enfermeira. Doutora em Enfermagem. Docente do NFR/UFSC. Florianópolis, SC. E-mail: janecanders@nfr.ufsc.br.

"IIEnfermeira. Doutora em Enfermagem. Docente do NFR/UFSC. Florianópolis, SC. E-mail: betinam@nfr.ufsc.br.

IV Enfermeira. Pós-Doutora pela Lawrence Bloomberg Faculty of Nursing at University of Toronto, Canada. Docente Associada do NFR/UFSC. Pesquisadora do CNPq. Florianópolis, SC. E-mail: padilha@nfr.ufsc.br.

${ }^{\vee}$ Enfermeira. Doutoranda do Programa de Pós-graduação em Enfermagem da UFSC. Florianópolis, SC.
}

\section{RESUMO}

O processo de enfermagem caracteriza-se como uma tecnologia de cuidado. Este estudo objetivou refletir sobre o ensino do Processo de Enfermagem no curso de graduação em enfermagem. Considera-se que o mesmo se configura como uma tecnologia leve-dura no cuidado de enfermagem, favorável a efetividade e eficiência dos serviços de saúde. A reflexão aponta três temas de discussão: o processo de enfermagem como referencial para a prática de enfermagem; o processo de enfermagem como tecnologia do cuidado e o seu ensino do nos cursos de graduação em enfermagem. Este é entendido como uma tecnologia leve-dura, pois, em sua concepção, os saberes estruturados, associados ao diálogo e a escuta sensível, estão presentes e definem a ação da enfermeira. Possibilita a construção de uma consciência crítica para os envolvidos no cuidado, sendo a comunicação um instrumento necessário. Surgem novos modos de trabalho e tecnologias que são incorporadas continuamente às práticas dos profissionais. Isto traz um desafio para a formação e prática das Enfermeiras, no qual se considere o novo saber, pois o processo enfermagem como tecnologia exige abertura para a complexidade e um exercício crítico para o atendimento das necessidades dos seres humanos, foco do cuidado de enfermagem.

Descritores: Educação em Enfermagem; Processo de Enfermagem; Tecnologia; Enfermagem.

\section{ABSTRACT}

The nursing process is characterized as a technology of care. This study aimed to reflect on the teaching of nursing process in the undergraduate program. Considering that one organizes oneself with a technology light-firm in the nursing care, suitable to effectiveness and efficacy of the health services. The reflection points out three topics for discussion: the nursing process as an indication to the usage of nursing; the nursing process as a care technology and its teaching in the undergraduate program. This is understood with a light-firm technology, because, in its conception, the structured knowledge, associated with dialogue and sensible hearing, they exist and define the nurse's action, allowing the building of a critical conscience to the people involved in the care, being communication a necessary instrument. New ways of work and technology emerge that are incorporated to the professional's practice. It brings a challenge to the nurse's formation and practice, which considers the new knowledge; because the nursing process as technology demands opening to the complexity and a critical exercise to the attention to the human beings necessities, focus of the nursing care.

Descriptors: Nursing Education; Nursing Process; Technology; Nursing.

\section{RESUMEN}

El proceso de enfermería se caracteriza por ser una tecnología de atención. Este estudio tuvo como objetivo reflexionar sobre la enseñanza del proceso de enfermería en el curso de pregrado en enfermería. Considerando que el mismo se configura como una tecnología leve-dura en el cuidado de enfermería, favorable a efectividad y eficiencia de los servicios de salud. La reflexión apunta tres temas de discusión: el proceso de enfermería como referencial para la práctica de enfermería; el proceso de enfermería como tecnología del cuidado y su enseñanza en los cursos de pregrado en enfermería. Este es entendido como una tecnología leve-dura, ya que, en su concepción, los saberes estructurados, asociados al diálogo y a la escucha sensible, están presentes y definen la acción de la enfermera, posibilitando la construcción de una conciencia crítica para los involucrados en el cuidado, siendo la comunicación un instrumento necesario. Surgen nuevos modos de trabajo y tecnologías que son incorporadas continuamente a las prácticas de los profesionales. Esto trae un desafío para la formación y práctica de las Enfermeras, en el cual se considere el nuevo saber, pues el proceso enfermería como tecnología exige apertura para la complejidad y un ejercicio crítico para atender las necesidades de los seres humanos, foco del cuidado de enfermería.

Descriptores: Educación en Enfermería; Procesos de Enfermería; Tecnología; Enfermería. 


\section{NTRODUÇÃO}

Os princípios filosóficos do Sistema Único de Saúde provocam mudanças nos cenários da prática quando, para atendê-los as instituições buscam novas maneiras de gerenciar e realizar o cuidado em saúde. A nova concepção de saúde influencia o processo de trabalho de todos os profissionais e resulta em um novo modelo de atendimento para a população, gerando não só expectativas positivas, mas também resistência destes frente à imposição das novas tecnologias e novos modos de trabalho. Isto passou a incluir a utilização de um saber específico, de constante aperfeiçoamento e atualização, que atendesse a velocidade de lançamento de novas tecnologias, visto que os conhecimentos técnico-científicos dos profissionais atuantes em ambientes tecnológicos desatualizam-se rapidamente. Isto pode ser observado no processo de produção da comunicação, das relações, de vínculos que conduzem ao encontro do usuário com necessidades de ações de saúde ${ }^{(1)}$.

O processo de trabalho na saúde é marcado pela ação do trabalho com o outro, pela alteridade. A enfermeira é reconhecida como figura central no processo de produção do cuidado, seja pelo vínculo que constrói com o paciente e sua família ou pela intermediação que faz com os demais profissionais ou pelos aspectos legais da profissão. Quando se fala de trabalho, vê-se que os trabalhadores envolvidos no processo de produção se posicionam no centro do debate e trazem a sua própria subjetividade para a ação. O processo de trabalho em saúde tem como finalidade a ação terapêutica de saúde; objeto - o indivíduo ou grupos de indivíduos com necessidades de saúde (afetadas ou não); instrumental - "os instrumentos e as condutas que representam o nível técnico do conhecimento que é o saber de saúde", ou seja, os saberes objetivados; e como produto final a própria prestação da assistência de saúde ${ }^{(2)}$

Neste sentido, entende-se que o cuidado, como foco da Enfermagem, é um processo interativo vivido no cotidiano e não se restringindo apenas a utilização de equipamentos e saberes estruturados, pois suas ações configuram-se como processos de intervenções, de relações e subjetividades. É nesse contexto que surge o processo de enfermagem como tecnologia do cuidado, pois embora tenha a mesma lógica de elaboração e execução, se apresenta como uma modalidade extremamente diversa, pois depende de quem o adota, do referencial teórico que o fundamenta e das perspectivas paradigmáticas dominantes neste ambiente de cuidado.

No entanto, na prática profissional, percebe-se que, nos cursos de graduação em Enfermagem, o ensino do Processo de Enfermagem não atende a esta expectativa, tendo em vista que provoca nos estudantes de Enfermagem sentimentos de insegurança e aversão e os docentes experimentam dificuldade e angústia no momento de ensinar. Além disto, no cotidiano dos serviços de saúde se observa a resistência das enfermeiras em implantar o Processo de Enfermagem quando as instituições preconizam sua aplicação ou não priorizam sua viabilização como atividade de rotina do trabalho de enfermagem. A partir destas constatações, questiona-se: como tem sido discutido o processo de enfermagem como tecnologia de cuidado na formação do enfermeiro?

Diante deste contexto, objetiva-se refletir sobre o ensino do Processo de Enfermagem, no curso de graduação em enfermagem, como tecnologia e ferramenta para a assistência. Justifica-se este estudo, pois o processo de enfermagem uma vez que o mesmo se configura como uma tecnologia levedura do cuidado de enfermagem, favorável a efetividade e eficiência dos serviços de saúde. Além disso, é uma possibilidade de reconhecimento e ampliação dos conhecimentos teóricos da enfermagem. Refletir sobre estas questões nos remete para três eixos de discussão: o Processo de Enfermagem como referencial para a prática de enfermagem; 0 processo de enfermagem como tecnologia do cuidado e o ensino do processo de enfermagem nos cursos de graduação.

\section{Processo de enfermagem como referencial para a prática de enfermagem}

A Enfermagem agrega ciência e arte na produção de conhecimentos necessários à sua realização na prática de cuidar das pessoas inseridas nos diversos grupos sociais. Sustenta-se em conhecimentos empírico, pessoal, ético, estético, educativo, político, científico e interdisciplinar, adequando sua ação às transformações sociais e comprometendo-se com a promoção e proteção à saúde, tanto no âmbito individual quanto coletivo. 0 modelo biomédico foi o pano de fundo para o nascimento e desenvolvimento da Enfermagem. Tal modelo vem sendo substituído pela noção de que o paciente deve ser compreendido em sua integralidade, despertando nas enfermeiras a busca de referenciais teóricos e modelos práticos que favoreçam a implantação de cuidados de enfermagem adequados ao processo de vida destas pessoas $^{(3)}$.

$\mathrm{Na}$ produção de conhecimento para a fundamentação da profissão, um marco importante é o aparecimento das teorias de Enfermagem, a partir da década de 50, nos Estados Unidos. As teorias fornecem subsídios para a construção de valores sobre o objeto, ferramentas para a intervenção na realidade, representando $o$ estado da arte profissional, referenciam o cuidado, têm potencial organizativo para o trabalho e estão propostas desde Florence Nightingale. Elas proporcionam o referencial 
teórico que norteia a implantação do Processo de Enfermagem, sob uma perspectiva de assistência sistematizada, havendo a retroalimentação entre o enriquecimento da teoria e o desenvolvimento da prática, refletindo satisfação na prática assistencial da enfermagem ${ }^{(4-5)}$. No Brasil, por volta dos anos 70, o marco de modernização para o ensino e prática de Enfermagem se fez com a publicação de Wanda de Aguiar Horta: Contribuições a uma teoria de Enfermagem. Desde então, o referencial vem sendo utilizado em muitos cursos de graduação em Enfermagem e também para auxiliar a sistematização da assistência em muitos hospitais, especialmente os universitários ${ }^{(6)}$.

Processo de enfermagem, metodologia da assistência e a própria sistematização da assistência ora apresentam-se como sinônimos e ora apresentam-se com diferentes definições. Diante dessa perspectiva considera-se que os termos têm significados distintos. Pode-se considerar que a SAE situa-se em uma esfera institucional, na qual a visão de organização das atividades de enfermagem interfere diretamente na implantação e implementação do processo de enfermagem e por assim dizer na metodologia subsidiada em uma teoria de enfermagem a ser utilizada na prática. Cabe destacar ainda que o processo de enfermagem é único, o que pode se modificar à luz do referencial teórico da unidade, é a metodologia da assistência de enfermagem proposta por cada uma das Teóricas de Enfermagem para o trabalho processual ${ }^{(7)}$.

Neste texto, discute-se o processo de enfermagem e espera-se que este seja uma forma reflexiva e crítica para a resolução das situações de saúde e doença das pessoas. Sua elaboração exige das enfermeiras a emissão de julgamentos pautados em conhecimentos biológicos, humanos, sociais, tecnológicos e éticos. Por outro lado, a lei do exercício profissional da Enfermagem 7498/86, em seu artigo 11, determina que cabe exclusivamente à Enfermeira a realização da consulta de enfermagem, da prescrição de enfermagem, dos cuidados diretos de enfermagem a pacientes graves, que regulamenta a implementação do Processo de Enfermagem à prática $^{(8)}$. O Processo de Enfermagem, embora a lei não explicite, é assumido como atividade específica da enfermeira e um modo de apresentar a cientificidade do trabalho desta profissional, pois o mesmo é dinâmico, aberto e contínuo, proporcionando à pessoa uma condição adequada para vivenciar de forma saudável o processo de saúde-doença.

O Processo de Enfermagem pode ser entendido como a aplicação teórica no cotidiano da assistência de Enfermagem. É a "dinâmica das ações sistematizadas e interrelacionadas, visando a assistência ao ser humano. Caracteriza-se pelo interrelacionamento e dinamismo de suas fases ou passos" $^{\prime(6)}$. Pode ainda ser compreendido como essência e instrumento da prática auxiliando a tomada de decisão, prevenindo e avaliando as consequências, sendo assim, é uma abordagem ordenada e sistemática da prática que procede de uma atividade intelectual deliberada ${ }^{(9)}$.

Seja qual for a definição dada ao processo de enfermagem, uma tríade estará sempre presente: o levantamento de dados, a análise e o plano de cuidados, os quais podem ser desenvolvidos em quatro fases: avaliação, identificação de problemas e/ou diagnóstico de enfermagem, intervenção ou implementação e evolução, embora o diagnóstico de enfermagem possa não ser mencionado e o plano de cuidados ser separado do processo de enfermagem.

O Processo de Enfermagem desperta a enfermeira para desenvolver a sua criatividade e sensibilidade, de forma ativa, sistemática e contínua, buscando sempre uma relação com a essência do ser humano, ao identificar os sinais enviados pelo corpo; observar o comportamento cotidiano das potencialidades e necessidades de ajuda de uma pessoa. Assim, identifica a quantidade e a qualidade de cuidado de Enfermagem que a situação requer para auxiliar na vivência do processo saúde-doença desta pessoa. Isto se justifica tendo em vista que os passos do processo remetem para o raciocínio científico, no qual a observação, a elaboração de hipóteses, a ação e avaliação são justificadas pelo entrelaçamento de diversos saberes que sustentam a escolha por este ou aquele cuidado. O Processo de Enfermagem é aplicável tanto ao indivíduo, quanto à família e aos grupos de pessoas, pois permite identificar as situações de saúde-doença vividas e sentidas pelo indivíduo ou pelo grupo de indivíduos; planejar, executar, avaliar e registrar a assistência de Enfermagem prestada cotidianamente.

O processo de enfermagem focaliza a reação da pessoa ao problema de saúde. Ao olhar para a pessoa, a enfermeira é capaz de identificar as necessidades e desejos e assim a investigação do corpo, da mente e do espírito maximiza a capacidade da pessoa para realizar ações importantes para ela. Também enfoca o ensino para o auto-cuidado, para a independência, envolvendo tanto a pessoa necessitada quanto a rede de pessoas significativas e de grupos da comunidade. Ainda podem ser listados como benefícios do Processo de Enfermagem o diagnóstico e tratamento de problemas de saúde (reais ou potenciais); o plano de cuidados eficaz e com redução dos custos (redução do número de internações e do tempo de internação); os registros que permitem auditoria e os estudos para o avanço da Enfermagem, promovendo o pensamento crítico, independente e flexível, que mantém o fator humano em vista(10). 
Além disto, a aplicação do processo de enfermagem exige capacitação contínua do Enfermeiro para o raciocínio clínico e utilização de ferramentas para o exame clínico. O pensamento crítico é deliberado, busca resultados a partir das necessidades da pessoa, da família e da comunidade, orientado por padrões profissionais e código de ética; como habilidade precisa ser desenvolvida a partir da prática questionadora, flexível e contextualizada(10).

Neste sentido, além das habilidades técnicas necessárias para a segurança do fazer, a enfermeira deve desenvolver as habilidades interpessoais. Abordar o paciente para uma investigação de seu estado atual, identificar problemas de enfermagem e estabelecer uma conduta terapêutica exige da enfermeira conhecimentos sobre comunicação terapêutica dentre outras técnicas para que ocorra a sequência de eventos mentais que permitem a elaboração e aplicação do processo de enfermagem. Vale destacar que a comunicação terapêutica para ser alcançada necessita que sejam desenvolvidos comportamentos que demonstrem confiança, respeito, envolvimento profissional. Assim, a enfermeira deve ser preparada para aceitar as pessoas, tendo em vista subjetividades, crenças e valores, rompendo uma prática mecanizada, preocupada em responder o que se espera que ela faça, sendo assim pobre em reflexões. A comunicação terapêutica aparece como uma possibilidade, pois a introspecção é um processo construtivo de aprendizagem que conduz a uma atitude orientada por princípios éticos universais e deve ser favorecida pelas instituições de saúde e de ensino no que se refere a promoção de cursos para este fim $^{(4,11)}$

Portanto, o processo de enfermagem permite o amadurecimento profissional, uma vez que alia sistematicamente dois instrumentos de trabalho, a observação e a comunicação, para a realização de suas etapas seqüenciais e interrelacionadas; objetivando resultados. Estas características o configuram como uma tecnologia leve-dura de trabalho, tema do próximo eixo de discussão.

\section{O processo de enfermagem como tecnologia do cuidado}

Tanto no âmbito da atenção primária a saúde quanto no hospitalar, a introdução contínua de produtos tecnológicos provoca modificações diretas sobre as relações entre as pessoas, profissionais ou pacientes, suplantando muitas vezes a sua presença.

A palavra tecnologia provém de técnica que significa arte ou habilidade, mostrando a tecnologia como atividade voltada para a prática: é o conhecimento científico transformado em técnica que por sua vez amplia a produção de novos avanços por meio do conhecimento científico. A técnica compreende um conjunto de regras que dirigem eficazmente uma atividade qualquer, capaz de produzir resultados em qualquer campo da atividade humana. O objetivo é aumentar a eficiência em todas as esferas, congregando diversos conhecimentos, sejam científicos, empíricos ou intuitivos, aplicáveis na produção e comercialização de bens e serviços. Desta forma, a enfermeira utiliza vários saberes científicos com o objetivo de encontrar a solução para problemas ou situações vivenciadas na prática, decorrentes do encontro entre ela e o usuário dos serviços de saúde, que pode se caracterizar como tecnologia de cuidado ${ }^{(3)}$.

Falar em tecnologia é falar em ação intencional sobre o mundo com vistas a produção de bens, tanto materiais quanto simbólicos. O trabalho em saúde opera tanto com tecnologia de relação, de encontro de subjetividades e simbólica quanto a tecnologia dura, por esta razão, há uma classificação em três categorias $^{(12)}$ : a) Tecnologia dura: representada pelo material concreto como equipamentos, mobiliário tipo permanente ou de consumo; b) Tecnologia levedura: incluindo os saberes estruturados representados pelas disciplinas que operam em saúde, a exemplo das clínicas médica, odontológica, epidemiológica, entre outras e; c) Tecnologia leve: que se expressa como o processo de produção da comunicação, das relações, de vínculos que conduzem ao encontro do usuário com necessidades de ações de saúde.

Os profissionais de saúde não se sentem competentes para discutir tecnologia em saúde. Enfatiza-se que discutir tecnologia não é discutir equipamentos, nem o moderno, nem o novo. Passa por "discutir o proceder eficaz de determinados saberes e suas finalidades"(13). A tecnologia em saúde está marcada pela conformação do que é saúde e doença, normal e patológico, vida e morte, além da conformação de determinado saber que permita aos profissionais ler e intervir nestes processos. Esta reflexão leva a perceber que as principais tecnologias que temos que usar estão centradas no conhecimento, ou seja, "é no nosso saber que temos o principal patrimônio para gerar opções tecnológicas de atenção a saúde ${ }^{(14)}$.

Pesquisa realizada aponta que a Enfermagem desenvolve tecnologias leves, tais como: a) Acolhimento, no preparo de um ambiente físico que proporcione prazer, conforto e bem-estar ao cliente, a despeito das características intrínsecas ao hospital enquanto ambiente frio e impessoal, a exemplo da decoração com flores, cores alegres e presença da música. Esse acolhimento proporciona a relação de ajuda-confiança, transmitindo confiança ao cliente. b) Interação, que se desenvolve através do diálogo; da escuta sensível; das conversas tanto formais, relativas às explicações e informações necessárias à 
realização de um procedimento técnico, quanto às informais; no colocar-se à disposição do outro; no atribuir importância às inquietações e questionamentos do cliente e sua forma de se ver e se conduzir no mundo; no respeito às suas crenças e valores; c) Expressão do afeto, demonstração de alegria, de carinho e do toque no desenvolvimento do cuidado ${ }^{(14)}$

Diante destas classificações, o Processo de Enfermagem pode ser entendido como uma tecnologia leve-dura, pois em sua concepção os saberes estruturados, associados ao diálogo e a escuta sensível estão presentes e definem a ação da enfermeira, possibilitando a construção de uma consciência crítica para os envolvidos no cuidado. Entretanto, há que se pensar que a comunicação é outro instrumento necessário para mediar a tecnologia do Processo de Enfermagem, pois é no diálogo que a subjetividade do paciente e a subjetividade da enfermeira se expressam.

O espaço concreto institucionalizado é dotado de razão e vontade e atende a princípios éticos, que permitem uma atitude consciente da enfermeira. Neste sentido, o risco da tecnologia ser justificativa última do cuidado não acontece, pois as questões do cotidiano humano são problematizadas e valorizadas conduzindo a ação dentro da aceitação mútua dos valores, crenças e conhecimentos que ambos, enfermeira, paciente e família, previamente já possuem. Trata-se de uma ação social, pois ocorre em função do comportamento e guarda uma relação reflexiva entre si e a orientação dos sujeitos envolvidos no encontro. Cria-se o espaço intersubjetivo, onde, as verdades de cada um se expressam e a enfermeira escolhe uma ação resolutiva e eficaz para a situação, baseada na sua experiência profissional e de vida, considerando a outra subjetividade ${ }^{(4)}$.

Embora o processo de enfermagem seja uma tecnologia leve-dura essencial para o cuidado de enfermagem, a dificuldade para seu ensino e operacionalização está presente no âmbito acadêmico e assistencial. A reflexão acerca dessa temática constitui o próximo eixo que aponta os impasses e perspectivas.

\section{O ensino do processo de enfermagem na graduação}

A reflexão que se faz premente neste momento é sobre a historicidade do processo de trabalho da enfermeira tendo como foco o processo de enfermagem. A forma processual de cuidar no Brasil surgiu a partir de uma lei que atendia a exigência do mercado político, econômico e financeiro e da iniciativa de um grupo de enfermeiras em uma época que a equipe de enfermagem estava em transformação e o número de enfermeiras no país era pequeno $^{(15)}$. Pode-se citar que na década de 80 cerca de $60 \%$ dos elementos da equipe de enfermagem atendentes de enfermagem e apenas $10 \%$ eram enfermeiras. Consequentemente, a força de trabalho nas unidades de saúde conduzida por enfermeiras tinha uma grande dificuldade em romper com as estruturas biomédicas para a implantação de um modelo de assistir contrário à prática vigente ${ }^{(2)}$.

Observa-se que, em meio a esta transição, o Processo de Enfermagem apareceu como mais uma obrigação tanto para a enfermeira da prática assistencial quanto para a da prática docente, embora tivesse como meta sustentar teoricamente o exercício profissional. Além disto, a existência de várias teorias de enfermagem possibilitava vários modelos de processo de enfermagem, cuja consolidação como marco acabou se perdendo, pois a compreensão e implantação do mesmo poderiam ser diferentes. Estas diferenças, ao longo do tempo foram se transformando em dificuldades para o ensino uma vez que não existia uma linguagem padrão, fruto de um consenso entre a docência e a prática.

Com a Teoria das Necessidades Humanas Básicas de Horta houve uma tentativa de uniformização no Brasil, mas os entraves relacionados a imagem coletiva do processo de enfermagem já estavam consolidados. O ensino e a prática do Processo de Enfermagem estavam ao mesmo tempo em fase de implantação, validação e modificação de sua operacionalidade. Esta dialética provocou nas pessoas, ao longo do tempo, cansaço e descrédito, exatamente porque seu potencial para a eficiência e efetividade ainda estava em formação e não se apresentava como algo delineado e claro.

Convém refletir que o processo ensinoaprendizagem inclui os atos de ensinar, de aprender e de apreender, que muitas vezes são dissociados, pois existe a ideia de que ensinar é apenas apresentar ou explicar um determinado conteúdo. Assim, o docente se preocupa com as técnicas de ensino, transformando a sala de aula em um espaço onde ele fala, com a intenção de despertar o acadêmico para o conhecimento. No entanto, o processo de ensinar, que se completa com o aprendizado e a apropriação pelo estudante do conteúdo ensinado, muitas vezes não acontece ${ }^{(16)}$. O resultado da aprendizagem vai além do ato de aprender, há necessidade de que o estudante segure, assimile mentalmente, ou seja, transforme o aprendizado em ato de apreensão.

Outro aspecto do processo de ensinar diz respeito ao envolvimento do docente com a área que ensina, isto é, quando o docente "saboreia, na lida cotidiana profissional e/ou na pesquisa, e a socializa com seus parceiros na sala de aula", o acadêmico se percebe incluído no saber, "um saber o quê, um 
saber como, um saber por quê e um saber para quê" ${ }^{\prime(16)}$.

O ensino do Processo de Enfermagem pode ser analisado tendo em vista a grade curricular do curso, a ótica do docente, do estudante, da instituição de ensino e dos serviços de saúde e deve ser iniciado quando o estudante já incorporou conhecimentos das ciências básicas e específicas da profissão. Com relação aos docentes, há necessidade de uma linguagem comum e convicção quanto a aplicação do Processo de Enfermagem, além de manterem a mesma postura e dominarem os conhecimentos que favoreçam o ensino do processo de enfermagem. Ainda é destacado que os estudantes devem aprimorar os conhecimentos e desenvolver suas habilidades para observar, analisar, investigar e, principalmente, terem interesse em apreender os assuntos abordados no ensino; institucionalmente precisa haver disposição de seus dirigentes e do corpo das enfermeiras para a implantação do processo de enfermagem.

Os recursos pedagógicos devem ser variados, utilizando aulas expositivas, discussão de casos clínicos e recursos computacionais, além disto, as instituições de saúde que recebem estudantes contribuem para o sucesso da aprendizagem quando empregam sistematicamente o Processo de Enfermagem. Permeando todo este contexto está a forma como o discente aborda o assunto, ou seja, para que o processo de ensino-aprendizagem seja efetivo há necessidade de que a comunicação tenha caráter interativo e se afaste do modelo vertical de ensinar. Assim sendo, são estabelecidas condições para o raciocínio clínico, crescimento profissional e pessoal evitando uma transmissão padronizada de assuntos que apenas informam ${ }^{(3)}$.

A compreensão da historicidade do processo de trabalho da enfermeira, a escolha e apreensão de uma teoria para nortear a operacionalização do Processo de Enfermagem podem constituir a base para encantar os docentes e os acadêmicos sob uma perspectiva elaborada e compreensiva.

No ensino-aprendizagem do processo de enfermagem, docentes, acadêmicos e enfermeiros se deparam com estas questões, acrescidas pela menor valorização da prática de cuidado sistematizado, diante da valorização das técnicas e procedimentos. Assim será preciso também discutir e reorganizar estes conhecimentos, pois as dificuldades e resistências continuarão existindo.

\section{REFLEXÕES FINAIS}

Pode-se inferir que o ensino do processo de enfermagem garante a formação de um profissional competente. Assim, seu ensino traz como desafio a capacitação dos graduandos e docentes de Enfermagem para a sua utilização como ferramentas que cria novas dimensões na prática profissional, delineando impasses e perspectivas.

Muitos são os desafios a serem superados e estratégias a serem utilizadas no ensino do Processo de Enfermagem. Torna-se necessário abrir espaços para a reflexão sobre os meios e fins da utilização do Processo de Enfermagem como fundamental ao cuidado nas práticas de saúde que viabiliza a reconstrução da intersubjetividade associada ao uso das demais tecnologias.

Tanto o acadêmico como o docente e o profissional encontram diante de si um legado de perguntas e de sentimentos que precisam ser desvelados, conhecidos e reconhecidos para que possam ser superados e a imagem coletiva do processo de enfermagem vigore como uma tecnologia leve-dura. Cabe uma ação conjunta entre a academia e as instituições de saúde para definir as estratégias de ensino de modo que os grupos de profissionais e estudantes sintam-se estimulados para sua apreensão e aplicação e assim incorporem a importância desta ferramenta para o seu cotidiano. A iniciativa para este empreendimento será daquele que estiver sensibilizado, mas se acredita que o ato de ensinar seja essencial para o sucesso desta jornada.

É fundamentalmente nessa perspectiva que se entende o lugar privilegiado do cuidado nas práticas de saúde, como uma atitude e espaço para relações humanas, de compartilhamento de saberes e não somente o desenvolvimento e aplicação de tecnologias em saúde.

Diante dos avanços tecnológicos e da conseqüente competitividade impõe-se a urgente renovação do conhecimento, pois o cuidado de saúde está cada vez mais complexo e exige profissionais capazes para sua realização, com conhecimento e desenvolvimento de habilidades. A resposta a estes desafios pressupõe o aprimoramento da atuação das enfermeiras dentro do sistema de saúde. Esta rápida evolução das tecnologias de saúde tem levado os profissionais de Enfermagem, em particular na área assistencial, a buscar um aprimoramento constante dos seus conhecimentos para atender às exigências crescentes do mercado de trabalho e do sistema de saúde vigente.

No entanto, evidencia-se que não só a sintonia com os enfermeiros dos cenários de prática, além de sua modernidade no sentido de integrar as mudanças na sociedade e nos setores de prática, como também de contribuir para a evolução conhecimento voltado para a promoção da saúde; para a diversidade cultural; para uma melhor relação custo-benefício e maior integração entre os níveis de atenção à saúde; assim como para o gerenciamento da informação e tecnologia correlata. 
Soma-se a isto a necessidade de formação e atualização constante de profissionais, para que estes usem novas tecnologias de prestação do cuidado de Enfermagem. O desenvolvimento de classificações dos fenômenos de Enfermagem se deslocou além da capacidade de acadêmicos individuais, incorporandose às atividades profissionais o Processo de Enfermagem, caracterizado pelo histórico, diagnóstico, prescrição e evolução.

Com certeza, ainda são muitos os desafios e estratégias a serem superadas no ensino do Processo de Enfermagem como tecnologia diante destes impasses e perspectivas. Como tecnologia em que forem evidenciados alguns componentes, como: o exercício da consciência crítica, o exercício da cidadania, o exercício da liberdade e o exercício da autonomia, ao mesmo tempo, tanto para o profissional como para a clientela.

\section{REFERÊNCIAS}

1. Guimarães R. Bases para uma política de ciência, tecnologia e inovação em saúde. Ciênc. saúde coletiva. 2004; 9(2):372-87.

2. Pires D. Reestruturação produtiva e trabalho em saúde no Brasil. 2ed. São Paulo: Annablume. 2008

3. Souza LNA. Perspectivas do agir comunicativo implícitas no discurso da Enfermagem. [thesis]. Florianópolis: Departamento de Enfermagem/UFSC; 2005. 289p.

4. Fawcet J. Contemporary Nursing Knowledge Analysis and evaluation of nursing models and theories. Philadelphia: Davis; 2005.

5. Kim S, Kollak I. Nursing Theories: Conceptual and philosophical fuoundations. 2 ed. New York: Springer Publishing Company; 2006.

6. Horta WA. Processo de Enfermagem. São Paulo: EPU; 1979.

7. Fuly PSC, Leite JL, Lima SBS. Correntes de pensamento nacionais sobre sistematização da assistência de enfermagem. Rev. bras. enferm. 2008; 61(6): 883-7.

8. Lei N. 7.498 de 25 de junho de 1986. Dispõe sobre a regulamentação do exercício da enfermagem e dá outras providências. Diário Oficial da União (Brasília). 1986 jun 25.

9. Foster PC, Janssens NP. Dorothea E. Orem. IN: George JB. Teorias de enfermagem: os fundamentos para a prática profissional. Porto Alegre: Artes Médicas Sul; 2000. p. 90-107.

10. Alfavaro-Lefevre R. Aplicação do processo de enfermagem: um guia passo a passo. 5. ed. Porto Alegre: Artes Médicas; 2005.

11. Baggio MA. O significado de cuidado para profissionais da equipe de enfermagem. Revista Eletrônica de Enfermagem [Internet]. 2006 [cited 2008 may 12];8(1):9-16. Available from: http://www.fen.ufg.br/revista/revista8_1/original_01 htm.

12. Merhy EE. Praxis en salud un desafío para lo publico. São Paulo: Hucitec; 1997.

13. Merhy EE. Em busca da qualidade dos serviços de saúde: os serviços de porta aberta paa saúde e o modelo tecno assistencial em defesa da vida. In: Cecílio LCO. Inventando a mudança em saúde. 3ed. São Paulo: HUCITEC, 2006; p. 117-160.

14. Silva DCS. Alvim NAT, Figueiredo PA. As tecnologias leves em saúde e sua relação com o cuidado de enfermagem hospitalar. Esc. Anna Nery Rev Enferm. 2008; 12(2): 291-8.

15. Kletemberg DF. A metodologia da assistência de enfermagem no Brasil: uma visão histórica [dissertation]. Curitiba (PR): Programa de PósGraduação em Enfermagem/UFPR; 2004. 105 p.

16. Anastasiou LGC. Ensinar, aprender, apreender e processos de ensinagem. In: Anastasiou LGC, Alves LP, organizadors. Processos de ensinagem na universidade: pressupostos para estratégias de trabalho em aula. Joinville (SC): UNIVILLI; 2003. p. 11-38.

Artigo recebido em 24.11.08.

Aprovado para publicação em 27.10.09.

Artigo publicado em 31.03.10. 